Journal of Machine Engineering, 2020, Vol. 20, No. 1, 34-42

ISSN 1895-7595 (Print) ISSN 2391-8071 (Online)

Received: 03 February 2020 / Accepted: 12 February 2020 / Published online: 05. March 2020

deep drawing, energy saving, environmental issue, FEA

\begin{abstract}
Alexander BROSIUS ${ }^{1 *}$
Ali MOUSAVI ${ }^{1}$
\end{abstract}

\title{
DEEP DRAWING WITH MACRO STRUCTURED FOR AN ENVIRONMENTALLY FRIENDLY PROCESS
}

\begin{abstract}
The efficient use of resources are becoming more important due to economic and environmental requirements. In widely used forming processes like deep drawing an avoidance of lubricants - namely dry deep drawing - is a key factor for saving resources. In this paper, a combined approach consisting of a macro-structured tool design and a special modified protective coating is presented. The combination ensures a stable process with a sufficiently wide process window under dry conditions. The absence of lubricants in critical areas has to be compensated by tetrahedral amorphous carbon ta-C films which is additionally structured by Direct Laser Interference Patterning (DLIP) for further improvement of the tribological performance.
\end{abstract}

\section{INTRODUCTION}

Sheet metal forming by deep drawing is widely used in a variety of industries. Because of the comparatively high contact pressure onto the sheet metal as well as on the tool surface a high performance lubrication system is needed usually. Therefore, in nearly all sheet metal forming processes, lubricants are used in order to reduce friction, to reduce tool wear, and also to protect semi-finished parts from corrosion. Lubricants are often harmful to health and pollutes the environment directly due to waste disposal and indirectly by the required energy for application and removal of the lubricant. Increasing efforts have been made to decrease the amount of mineral oil used or to replace it with environmentally friendly lubricants [1]. Chlorine-, amine- and bond rite-free lubricants, and closed systems of lubrication and cleaning are practically discussed as feasible alternatives [2]. Nevertheless, the complete avoidance of lubricant would be the best variant. This is also the case under economic aspects, like Vollertsen et al. showed in [3]. Applying mineral oil based lubricants leads to additional production steps, because a post-process is required for cleaning the workpiece by solventbased agents. Therefore, from both economic as well as ecological point of view, a huge interest of a completely lubricant free deep drawing processes exists. In this work, an

\footnotetext{
${ }^{1}$ Chair of Forming and Machining Processes, TU Dresden -Institute of Manufacturing Technology, Dresden, Germany

* E-mail: Alexander.Brosius@tu-dresden.de https://doi.org/10.36897/jme/118216
} 
optimization of the tool design for deep drawing applications is suggested by employing functional coatings in combination with a macro-structuring to control friction, wear and material flow. As protective coating, a layer of tetrahedral amorphous carbon (ta-C) is applied which combines a low friction coefficient with a high hardness and wear resistance [4]. The ta-C coating is capable of taking over the tribological functions of the lubricant while protecting the microstructures [5]. The modification of the tool-namely the macro-structured tool surface - relates to the influence of reduced contact areas and the effect of alternating bending and restraining onto the springback behaviour of sheet metal parts. In the following the variant of a lubricant free deep drawing process is described and designed by means of numerical simulations and experimental investigations. The adapted macro-structured tool will ensure a sufficient process with beneficial product properties.

\section{METHODICAL APPROACH}

In case of a lubricant free deep drawing process, the friction forces will be larger compared the conventional setups. As a result, there is a high risk of material failure in the bottom area of the part during drawing. To overcome this drawback, the acting friction forces in the flange area and at the die radius should be reduced to ensure process feasibility. The approach for minimisation of the friction forces during the process presented here is based on a macro structuring of flange area, a beneficial coating, and a laser micro structuring of the coated die radius.

\subsection{MACRO STRUCTURING OF FLANGE AREA FOR FRICTION REDUCTION}

The most promising method to reduce the friction in flange area is the usage of macrostructured deep drawing tools [6] because of its simplicity and its high capability to achieve this auim. In [7] it is shown that decreasing the friction forces for a defined friction coefficient, either the contact pressure or the contact area has to be reduced. The last point can be done easily by means of macro-structured tools. Nevertheless, minimizing the contact area will increase the risk of wrinkling because tangential compressive stresses in the plane of the sheet metal will cause buckling behaviour, which is usually avoided by a blankholder force. This effect is reduced by increasing the geometrical moment of inertia of the sheet metal.

During the drawing process, this is achieved by a small immersion of the blankholder into the drawing die, subsequently causing an alternating bending-unbending mechanism in the flange area. Similar to draw beads but as a side-effect, this deformations process can be used for the control of the material draw-in. The geometry of macro structures, the wave length $\lambda$ and peak-to-valley height difference $\delta$ are two variable process parameters which are used to ensure a stable process. Figure 1 shows schematically the conventional deep drawing and the macro-structured process and illustrates the bending mechanism during the process as well as the reduced friction force due to decreased contact area. 

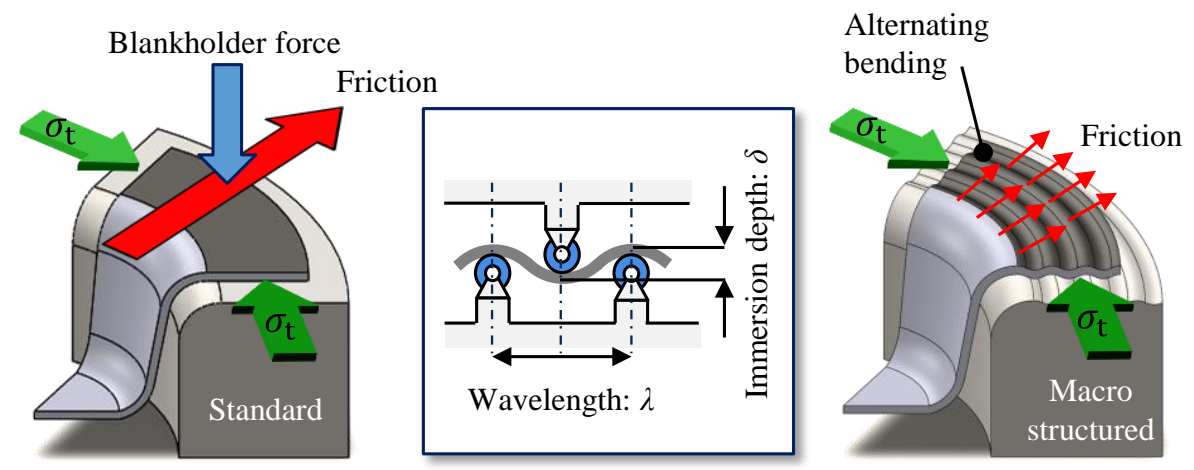

Fig. 1. Macro structuring of deep drawing tools to minimise the contact area and increase the process limits

As a consequence of this quite simple geometrical modification, deep drawing tools can reduce the contact area up to $80 \%$ to $95 \%$ depending on the structure that is applied. The reduction of the required blank holder force reaches values up to $90 \%$ compared with a standard tool design. As mentioned before, it also increases the resistance of the sheet metal against wrinkling and it allows the control of the material flow by adjustment of the immersion depth. [7] Obviously, a smaller immersion depth and higher wavelength induce larger radii in sheet metal, leading to decreasing buckling stiffness in the flange area. As a result, the risk of wrinkling increases. Oppositely, a large immersion depth and a smaller wavelength generates small radii in the sheet metal followed by an increasing deep drawing force, which in turn can cause bottom cracks immediately. As a result, the wave length and immersion depth should has to be determined with respect to material behaviour, especially the hardening behaviour and the material flow in the flange area.

Fig. $2 \mathrm{~b}$ shows the effect of the macro-structuring of the tools surface for a simulated 3D part geometry (Fig. 2a). Obviously the contribution on the punch force due to friction can be largely reduced. Therefore, the overall required force is decreased about $40 \%$. The best case would be a situation without any frictional forces, which can be emulated by finite-element analysis. For this optimal but only theoretical condition, the macro-structuring with frition is only slightly higher than the condition without friction.
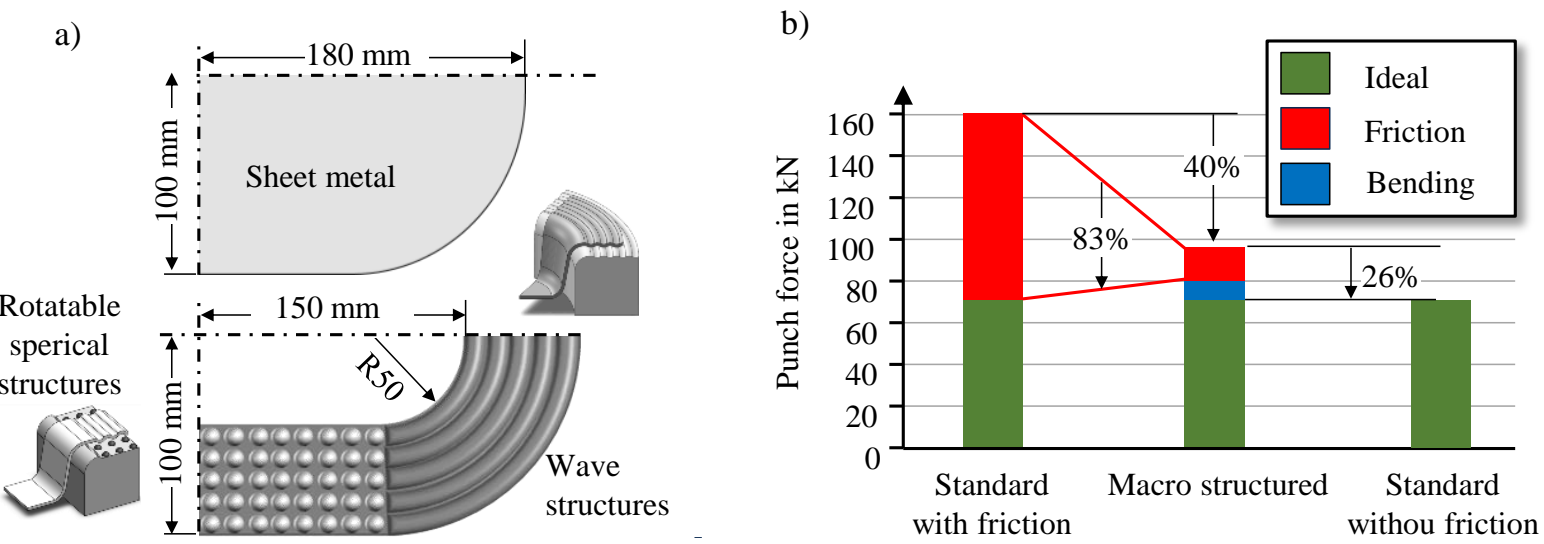

Fig. 2. a) Macro structured deep drawing tool; b) Difference between standard and macro structured deep drawing process regarding the punch force [6] 
To ensure time efficient identification of the relevant parameters, an analytical model was developed by Brosius et al. in [6] where the analytical and numerical results exhibit a very good agreement with the experimental tests. Within the scope of this model, the bottom crack and wrinkling can be predicted preliminarily using the energy method and stability analysis respectively. Here, the punch force is driven by total energy, as a sum of the singe energies shown in Fig. 3.
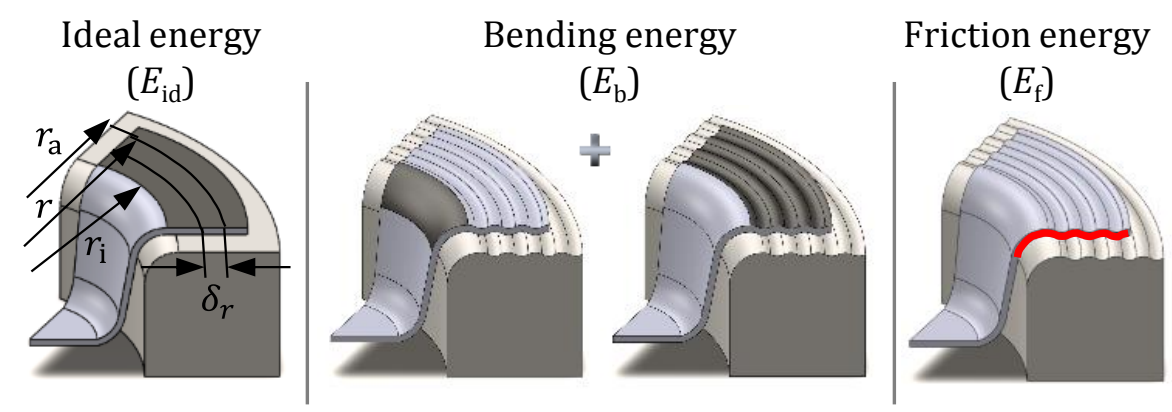

Fig. 3. Superposition method for energy calculation [7]

To predict wrinkling, a buckling analysis has been carried out to determine the critical tangential compressive stress as a function of sheet thickness, material properties and input parameters (immersion depth and wavelength). Only the most critical region of the sheet metal has to be considered here, because wrinkling starts at the weakest region in the part here the outer radius in the flange, see Fig. 4.

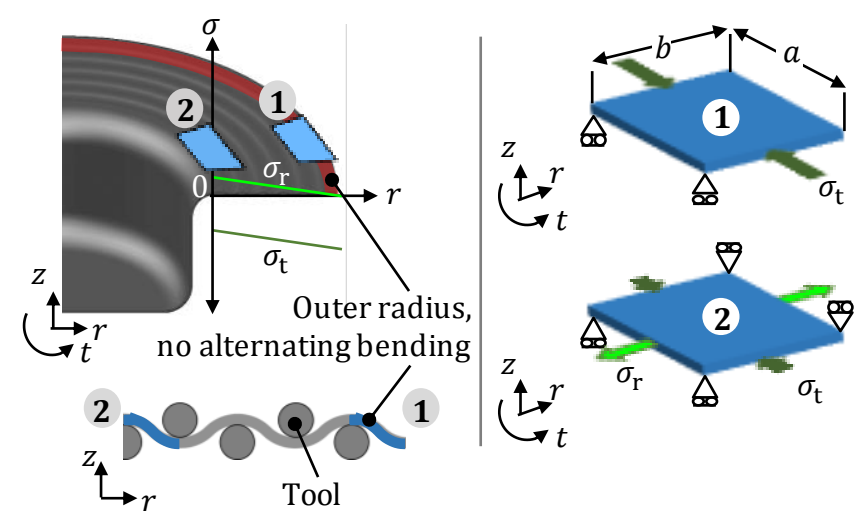

Fig. 4. Stress states of critical sheet metal areas for buckling analysis [7]

\section{2. ta-C COATING FOR FRICTION REDUCTION AT THE DIE RADIUS}

Amorphous carbon films are of interest in research as well as in industrial applications due to their remarkable properties like hardness and wear resistance [8]. Using this type of coatings, the adaption of the layer properties regarding friction reduction and high wear 
reduction can easily be done [9]. One well known representative is given by diamond-like carbon coatings (DLC), which can be produced by different process technologies. In PVD processes, the carbon source consists of graphite. If the graphite is evaporated in an ultra-high vacuum condition, the resulting coating is a pure carbon layer. Such layers consists of predominantly $\mathrm{sp}^{3}$-bonded carbon are known as tetrahedral amorphous carbon (ta-C). ta-C layers combine a low friction coefficient and anti-adhesive behaviour with a high hardness and wear resistance. Due to that, it can be used at the die radius of a macro structured deep drawing tools.

\subsection{DLIP STRUCTURING OF TA-C FILMS FOR ENHANCED TRIBOLOGICAL PROPERTIES}

The used ta-C film can be micro structured by Direct Laser Interference Pattering (DLIP) in order to modify the tribological performance e.g. by reducing the interfacial contact area. The DLIP processing is performed with a pulsed nanosecond solid-state laser system in a two-beam setup [10]. The laser beam is divided into two sub-beams by a beam splitter. Then, the sub-beams overlap on the sample surface with a specific overlapping angle, which in combination with laser wavelength determines the spatial period of the interference pattern (see Fig. 5). During DLIP structuring of the observed ta-C layer, a local $\mathrm{sp}^{3}$ to $\mathrm{sp}^{2}$ transformation takes place, which leads to a local decrease in atomic density associated with a localised surface swelling of the ta-C layer. Consequently, a line-like structure of sp $^{2}$-rich elevations is introduced at the surface of the ta-C layer.

a)

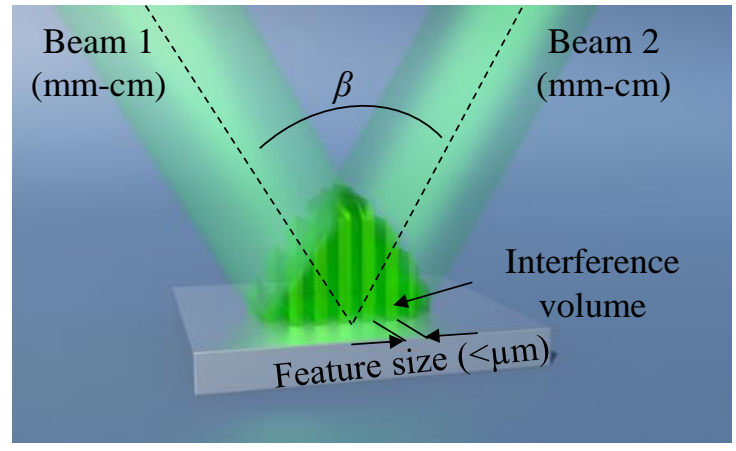

b)

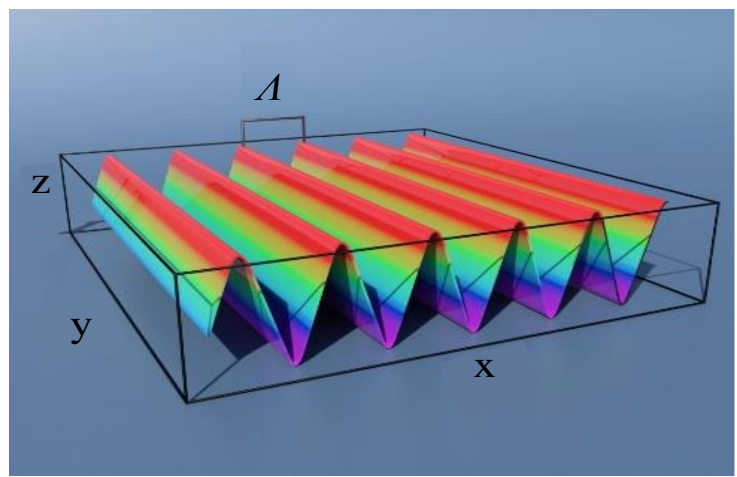

Fig. 5. a) Schematic representation of a 2-beam DLIP setup; a) Schematic representation of the interference pattern caused by 2D-DLIP [6] 
Here, the chosen structure period should be high enough to avoid a pronounced thermal diffusion from the interference maxima to the interference minima positions resulting in a complete $\mathrm{sp}^{3}$ to $\mathrm{sp}^{2}$ transformation at the surface of the ta-C film. Consequently, the DLIPstructuring introduces a periodic variation of $\mathrm{sp}^{3}$ - and $\mathrm{sp}^{2}$-rich regions which can improve the tribological properties of ta-C layer during the deep drawing process.

\section{RESULTS AND DISCUSSION}

\subsection{RESULTS OF MODELLING AND SIMULATION}

In order to verify the idea, analytical and numerical simulations as well as experimental analysis are done. The numerical results are based on a FEM-simulation (simufact.forming v13.1) using solid-shell elements with five integration layers over the thickness and a reduced integration scheme. Due to its suitability for deep drawing, the mild steel DC04 (1.0338) is chosen for this investigation. For the numerical and experimental analysis a macro structured axisymmetric drawing die with an inner radius of $50 \mathrm{~mm}$, an edge radius of $10 \mathrm{~mm}$ and a constant wavelength of $8 \mathrm{~mm}$ is utilized. Here, the immersion depth can be adjusted using distance rings from $0 \mathrm{~mm}$ to $0.4 \mathrm{~mm}$. Furthermore, rectangular macro structured deep drawing tools $(85 \mathrm{~mm} \times 85 \mathrm{~mm})$ with a corner radius of $20 \mathrm{~mm}$ are investigated. The die edge radius and wavelength of these tools are also $10 \mathrm{~mm}$ and $8 \mathrm{~mm}$ respectively. Due to other stress conditions, the immersion depth can vary from $0 \mathrm{~mm}$ to $1.0 \mathrm{~mm}$. Figure 6 shows typical examples of a successful and a failed process in order to demonstrate the feasibility of the process.

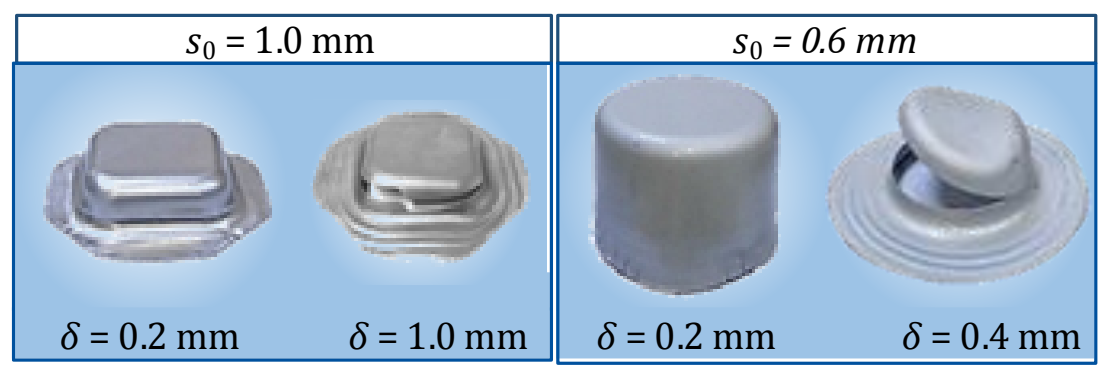

Fig. 6. Deep drawn parts (DC04, 1.0338) with macro structured tools [7]

To evaluate the analytical model for determination of the required energy in case of an axis-symmetric geometry, initial sheet diameter of 180 and $200 \mathrm{~mm}$ with an initial thickness of $0.6 \mathrm{~mm}$ and $1.0 \mathrm{~mm}$ are chosen. The analysis is done with two different immersion depths of 0.2 and $0.4 \mathrm{~mm}$.

Figure 7 shows the simulation results of different modelling strategies. The model based on analytical and numerical approaches are in good agreement with the experimentally obtained results. The scatter in the figure shown is quite small and was determined based on 
five repetitions per experiment. Despite the fact, that bottom cracks are not predictable by the analytical model, the modelling is powerful to determine the required energy and by this the punch force as a function of drawing depth.

Figure 8 shows the calculated results for a rectangular geometry, which are also in good agreement with the experimental observations. Obviously, the analytical determined energies are always lower than the experimentally measured energy because the shearing in the surrounding of the corner areas is unaccounted in the modelling. Despite this simplification, the quality of the achieved results is good and can be used to determine the influence and the sensitivity of the wavelength and the immersion depth onto the total energy and the punch force.

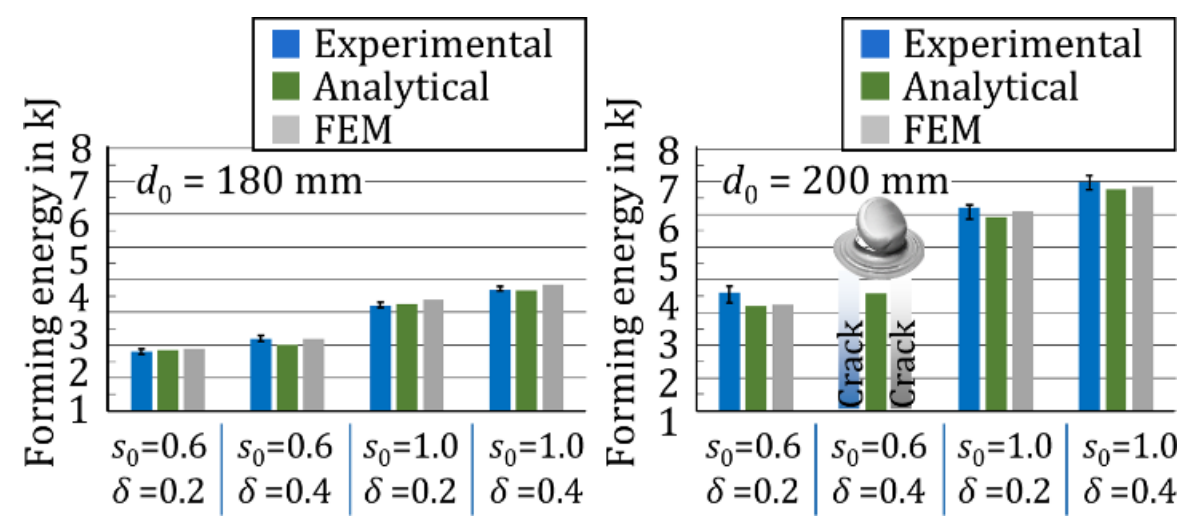

Fig. 7. Comparison between different modelling strategies for an axisymmetric geometry [7]

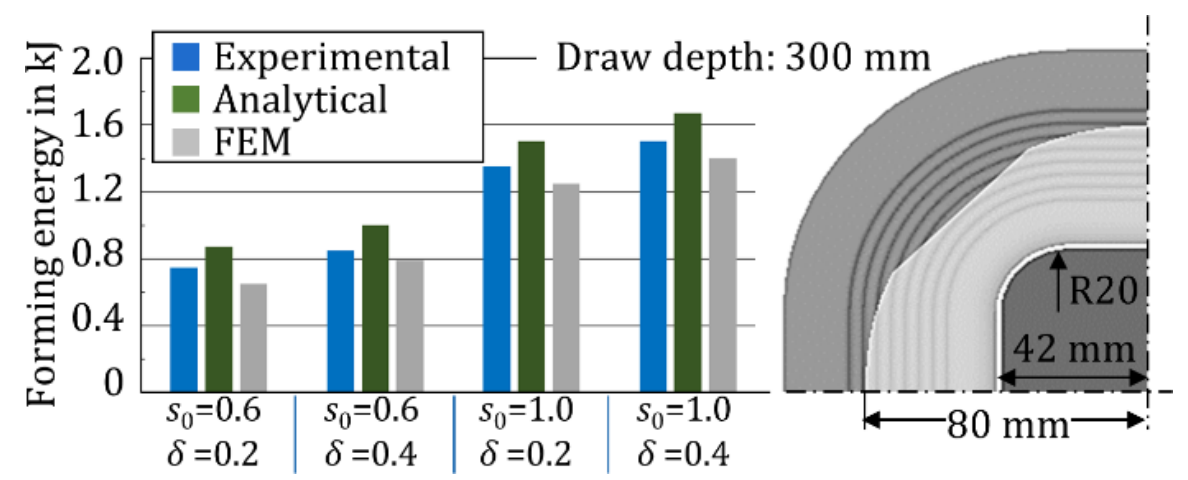

Fig. 8. Comparison between different modelling strategies for a rectangular geometry [7]

\subsection{DLIP STRUCTURING OF TA-C FILMS FOR ENHANCED TRIBOLOGICAL PROPERTIES}

Figure 9a shows the effect of the microstructuring of the coated tool surface. The uncoated tool shows the highest friction coefficient. By using an commercial lubricant (company WISURA, type ZO 3368), the friction coefficient can be reduced to 0.21. Coating the tool with a ta-C film reduces the friction coefficient to approximately 0.17 . A draw-bend tests of the coated and DLIP-structured tool show a comparable but slightly increased friction coefficient ( 0.18). However, an analysis of the unstructured and DLIP-structured ta-C coated tools after the draw-bend tests by means of white light interferometry indicates a significantly 
reduced amount of worn material ( $90 \%)$, as shown in Fig. 9b. This can be attributed to the modified $\mathrm{sp}^{3} / \mathrm{sp}^{2}$ ratio at the interface where $\mathrm{sp}^{2}$-rich elevations lead to a change in the contact geometry. Furthermore, the $\mathrm{sp}^{2}$-rich regions can act as an enhanced shear interface accompanied by shear localisation, which in turn acts as a protection of the underlying ta-C surface.
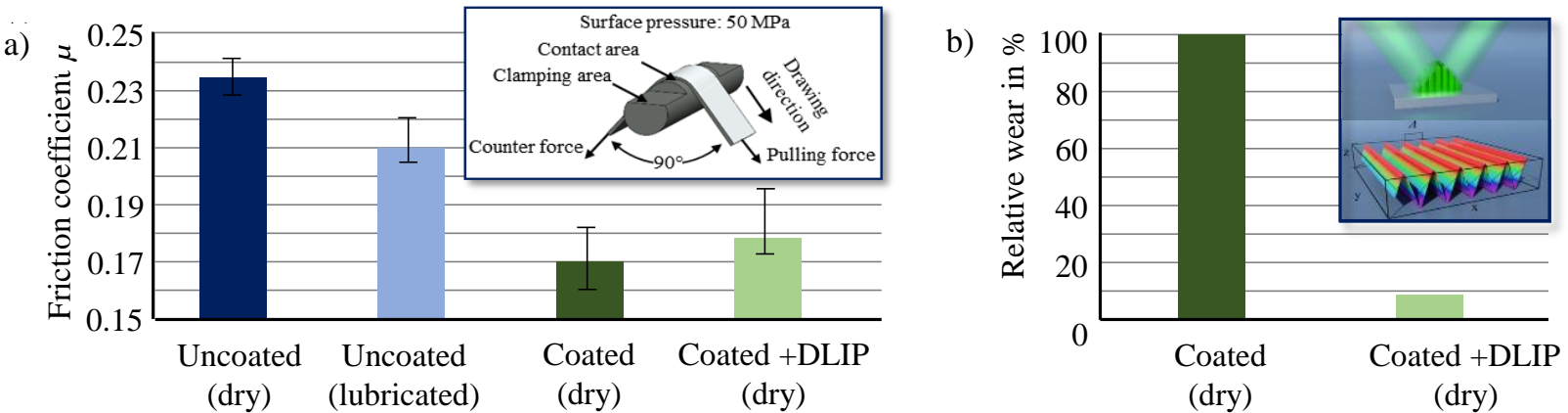

Fig. 9. a) Friction coefficient from draw-bend test; b) Comparison between ta-C coated and micro structured ta-C coated tool regarding wear [6]

\section{SUMMARY}

Macroscopic and microscopic approaches are presented in order to enable deep drawing as a lubricant free forming process. Two flexible parameters are introduced in order to achieve this task: the wavelength of the macro structure and the immersion depth of the blankholder. The simulation shows a significant reduction of friction forces with macro structured tools (up to 83\%). Moreover, a ta-C coating on the die radius also reduces the friction coefficient up to $20 \%$ compared to lubricated conditions. Micro structuring the ta-C layer with DLIP technology has no significant effect on the friction coefficient, but reduces the wear of the layer down to $10 \%$. Future studies will focus on complex 3D-geometries and other materials like aluminium. With the fundamental knowledge about the relevant acting effects, it is possible to consider more complex geometries, which are typical for industrial application.

\section{ACKNOWLEDGEMENT}

The German Research Foundation (DFG) is greatly acknowledged for financial support (Priority Programme 1676 "Dry Metal Forming - Sustainable Production through Dry Processing in Metal Forming " - SPP 1676). The authors would like to thank the Chair of Large Area Laser Based Surface Structuring for creating the coatings and the DLIP structures.

\section{REFERENCES}

[1] TAKEUCHI M., KASHIMURA F.N., 1999, Development of environmentally friendly lubricant with high performance and simple treatment for cold forging, Proceedings of the 6th ICTP, 383-390.

[2] K. TAMAOKI K., MANABE K-I., KATAOKA S., AIZAWA T., 2010, Electroconductive ceramic tooling for dry deep drawing, Journal of Materials Processing Technology, 210, 48-53. 
[3] VOLLERTSEN F., FLOSKY H., SEEFELD T., 2015, Dry Metal Forming - a Green Approach, 60 Excellent Innovations in Metal Forming, Springer Vieweg, ISBN 978-3-662-46311-6, 113-118.

[4] ROBERTSON J., 2002, Diamond-like amorphous carbon, Material Science and Engineering, 37, 129.

[5] MOUSAVI A., SCHOMÄCKER M., BROSIUS A., 2014, Macro and micro structuring of deep drawing's tools for lubricant free forming, Procedia Engineering, 81, 1890-1895.

[6] MOUSAVI A., KUNZE T., ROCH T., LASAGNI A., BROSIUS A., 2017, Deep drawing process without lubrication - an adapted tool for a stable, economic and environmentally friendly process, ICTP 2017, 48-53.

[7] BROSIUS A., MOUSAVI A., 2016, Lubricant free deep drawing process by macro-structured tools, CIRP Annals - Manufacturing Technology, 65, 253-256.

[8] CHOWAlla M., ROBERTSON J., CHEN C., SILVA S., DAVIS C., AMARATUNGA G., MILNE W., 1997, Influence of ion energy and substrate temperature on the optical and electronic properties of tetrahedral amorphous carbon (ta-C) films, J. App. Phys., 81, 139-147.

[9] FIELD S.K., JARRATT M., TEER D.G., 2004, Tribological properties of graphite-like and diamond-like carbon coatings, Tribology International, 37, 949-956.

[10] ROCH T., BEYER E., LASAGNI A., 2010, Surface modification of thin tetrahedral amorphous carbon films by means of UV direct laser interference patterning, Diam. Relat. Mater., 19, 1472-1477. 